\title{
Surgimento e Evolução do Cromatógrafo Líquido de Ultra Alta Eficiência(UPLC), sua Aplicabilidade, Vantagens e Desvantagens
}

\section{Emergence and Evolution of the Ultra High Performance LiquidChromatograph (UPLC), its Applicability, Advantages and Disadvantages}

\author{
Artur Eduardo Alves de Castro*a; Roberto da Silva Gusmão; Dirceu Aparecido Gonçalves de Souza; Honoria Paula \\ Alves de $\mathrm{Sa}^{\mathrm{a}}$; Regina Mara Silva Pereira ${ }^{\mathrm{a}}$; Márcio Luiz dos Santos ${ }^{\mathrm{a}}$
}

aUniversidade Anhanguera de São Paulo, Programa de Pós-Graduação Stricto Sensu em Biotecnologia e Inovação em Saúde. SP, Brasil.

*E-mail: aeducastro@hotmail.com

\begin{abstract}
Resumo
A cromatografia líquida convencional é uma técnica analítica que consome uma grande quantidade de solventes orgânicos, comprometendo parte significativa dos gastos com laboratórios de controle de qualidade, desenvolvimento analítico e de estabilidade nas indústrias farmacêuticas, que levou os pesquisadores a buscarem meios e alternativas que pudessem mitigar este impacto econômico e ecológico. O Cromatógrafo Líquido de Ultra Alta Eficiência ou Ultra-High Performance Liquid Chromatography - UHPLC ou UPLC - foi uma alternativadesenvolvida com esses propósitos, alternativamente às análisescromatográficas convencionalmente executadas por aparelhos de Cromatografia Líquida de Alta Eficiência ou High Performance Liquid Chromatograph - HPLC. O objetivo do trabalho é traçar um histórico da evolução do UPLC, que possibilitou o melhoramento da técnica cromatográfica de alta eficiência, quanto ao tempo de análise e, consequentemente, redução do uso de solventes a partir dos princípios do HPLC, técnica atualmente utilizada com a maior quantidade de equipamentos instalados nos centros de pesquisa e universidades, além de laboratórios de rotina, de pesquisa e desenvolvimento das indústrias farmacêuticas em todo o mundo. Os resultados alcançados em relação à precisão analítica equivalente ou superior, redução do tempo de análise, redução do consumo de solventes orgânicos e, consequentemente, redução considerável do custo de análises quando comparados à Cromatografia Líquida de Alta Eficiência convencional, garantiram o sucesso da implantação desta técnica analítica, que vem ganhando espaço considerável nesses ambientes.
\end{abstract}

Palavras-chave: UPLC. UHPLC. HPLC.

\begin{abstract}
Conventional liquid chromatography is an analytical technique that consumes a large quantity of organic solvents, compromising a significant part of the costs in quality control laboratories, analytical development and stability in pharmaceutical industries, which led researchers to search for ways and alternatives that could mitigate this economic and ecological impact. The Ultra-High Performance Liquid Chromatography - UHPLC - (or UPLC - Ultra Performance Liquid Chromatography) - was an alternative developed for these purposes to the chromatographic analyses conventionally performed by High Performance Liquid Chromatography - HPLC devices. The objective of this study is to trace a history of the UPLC evolution, whichenabled the improvement of the high efficiency chromatographic technique, regarding the time of analysis and, consequently, reduction of the use of solvents from the HPLC principles, technique currently used with the largestamount of equipment installed in research centres and universities, besides routine laboratories, research and development of pharmaceutical industries around the world. The results achieved regarding the equivalent or superior analytical precision, reduction of analysis time, reduction of organic solvents consumption and consequently considerable reduction of theanalysis cost when compared to conventional High Performance Liquid Chromatography, guaranteed the success of the implementation of this analytical technique, which has been gaining considerable space in theseenvironments.
\end{abstract}

Keywords: UPLC. UHPLC. HPLC.

\section{Introdução}

O uso de solventes orgânicos em análises químicas de fármacos ocorre, principalmente, com associação entre polaridade do analito e do solvente utilizado, de forma a melhorar a dissolução, reduzindo o risco de parte do analito ficar insolúvel resultar em análises de teor com resultados duvidosos.

As técnicas analíticas de quantificação se baseiam na utilização de espectrofotômetros, as quais estão baseadas na determinação de teor frente aos padrões analíticos que consistem em matérias-primas purificadas e quantificadas de forma a oferecer ao laboratório uma referência que possa ser utilizada como padrão na avaliação do teor de matériasprimas na produção de medicamentos em escala industrial ${ }^{1}$. Com o passar do tempo, houve a necessidade de verificações periódicas de pureza (teor, umidade, etc.) em função de processos de degradação decorrentes de: hidrólise pela umidade do ambiente de armazenamento ou "headspace" (o espaço entre a superfície do líquido e sua tampa) do recipiente, decomposição química por acomodação molecular (formação de isômeros, misturas racêmicas ou torções estruturais moleculares), impurezas residuais não retiráveis, interações com os componentes de embalagem, condições especiais de 
armazenamento inadequadas (baixa umidade ou temperatura controlada) e modificações outras decorrentes do tempo de prateleira (shelflife) obrigando a estabelecer a tarefa de rotular o prazo de validade adequado à produção e ao consumo desses princípios ativos (SINDUSFARMA, 2015).

Os métodos cromatográficos restringiam a quantificar a pureza da matéria-prima e o teor final dos produtos farmacêuticos obtidos. Quando começaram a surgir problemas de teor de princípios ativos fora das faixas de especificação do produto, as pesquisas se concentraram para a identificação das substâncias que não respondiam pelo teor do ativo que poderiam se apresentar em quantidades significativas no produto final, podendo provocar aparecimento de efeitos adversos indesejáveis aos consumidores daqueles medicamentos. Assim, os estudos de estabilidade se tornaram relevantes na identificação dossubprodutos e controle de suas quantidades residuais. Novas técnicas analíticas começaram a ser aprimoradas para separação dos componentes principais e secundários de cada matéria-prima, frente às variáveis desde a rota de síntese, passando pelo armazenamento, até sua transformação em medicamento e chegando à destinação final dentro de um prazo útil de utilização: a validade do produto final (SINDUSFARMA, 2015).

A partir de então, a linha de raciocínio analítico começou a enxergar o analito como sendo uma mistura e não mais uma substância perenemente pura.

O uso da cromatografia tem registros entre 77 e 79 d.C. pelo historiador romano Caio Plínio II, que descreveu a técnica aplicada sobre papiro em sua grande obra de 37 livros, intitulada: "Naturalis História". Em 1800, Berzelius, estudando águas minerais para inúmeros tratamentos de saúde, descreveu a desmineralização por meio de filtração em areia. Em 1866, Friedlib Ferdinand Runge publicou várias imagens formadas em papel de filtro, a partir de soluções coloridas. Porém, o criador do termo "cromatografia", do grego: "croma" (cor) e "graph" (escrita), Mikhail Semenovich Tswett, empregou a técnica pela primeira vez em seus estudos e experimentos de separação de clorofilas (Figura 1), utilizando uma coluna de vidro contendo Inulina em pó (a fase estacionária que foi a melhor que ele encontrou após várias tentativas com outras), pela qual passou sua fase móvel hidroalcoólica (eluente), contendo as suas misturas que se separavam em cores diferentes e eram coletadas individualmente ao final do processo.
Figura 1 - Reprodução parcial. Esquema desenvolvido por Tswett em 1906

(a)

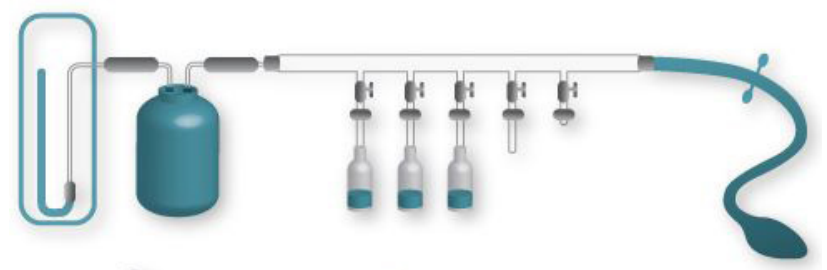

(b)

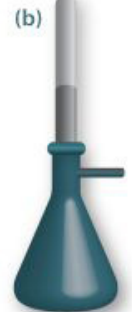

(c)

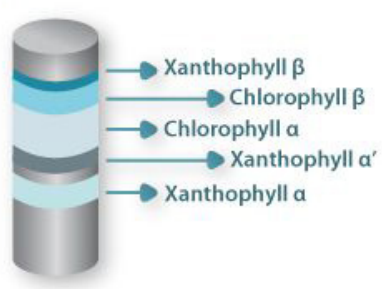

Fonte: Pacheco et al. (2015)

Em 1910, Tswett publicou o livro "Clorofilas no mundo vegetal e animal" e revolucionou tardiamente sua técnica no mundo científico (PACHECO et al., 2015).

O livro foi escrito em russo, idioma que nem ele mesmo dominava, cuja tradução era inacessível para boa parte dos pesquisadores. Eles não conseguiram compreender com clareza o método, o que os levou a reproduzir, de maneira errada, o que foi descrito. Tswett foi prejudicado por conta do advento da primeira Guerra Mundial: sua descoberta foi sabotada.

Além disso, sofreu preconceito dos pesquisadores químicos da época, pois estava propondo uma grande inovação na química analítica. Havia um propósito de isolamento de grandes proporções de princípios ativos principais e não de todos os componentes como a técnica de Tswett propunha. Isso adormeceu sua técnica cromatográfica por bastante tempo (PACHECO et al., 2015). Tswett foi redescoberto por estudiosos da química orgânica no final dos anos 1920 graças ao interesse destes por produtos naturais. Um deles, a pesquisadora Katharine Hope Coward conseguiu reproduzir os experimentos de Tswett em seus estudos sobre carotenoides, obtendo os mesmos resultados de separação de componentes eluídos em carbonato de cálcio. Porém, a cromatografia de coluna não teve reconhecidasua importância na época diante do advento da cromatografia plana em vidro e em papel.

Em 1938, surgiu na Rússia a cromatografia de camada delgada. Placas de vidro com um depósito na superfície de substância adsorvente (amido, por exemplo, depois sulfato de alumínio e, atualmente, sílica). Hoje em dia as cromatoplacas são comercializadas com placas de vidro e uma fina camada desílica ou placas de alumínio recobertas com sílica (PACHECO et al., 2015).

As técnicas cromatográficas são empregadas de várias maneiras: sobre placas recobertas com sílica, papel, em colunas e, também, passaram a ser automatizadas em equipamentos que transformaram as colunas de vidro (excessivamente 
grandes)em colunas de aço menores e pressurizadas contendo diferentes "recheios", a serem empregados em diversas técnicas de separação, sempre utilizando o princípio das diferenças de polaridade entre as várias substâncias e solventes ou misturas.

No ano de 1941 foi publicado um artigo em que os pesquisadores Archer John Porter Martin e Richard Lawrence Millington Synge descreveram uma nova forma de separação, denominada partição. Eles saturaram sílica com água de tal forma a ser empregada como fase estacionária para a separação de aminoácidos hidrolisados de proteínas. Assim, a fase estacionária passaria a ser a água, cujo suporte era a sílica e a fase móvel seria um líquido imiscível na água, em que o processo de partição ocorreria pelas fases líquidas e não mais por adsorção (MARTIN; SYNGE, 1941).

Para superar a dificuldade de visualização das separações de aminoácidos, que eram incolores, os pesquisadores colocaram indicadores que, misturados previamente à água, proporcionavam a visualização da separação por colorações distintas. Foi uma solução eficiente para a separação de aminoácidos hidrolizados de proteínas. Também evidenciaram que poderiam separar substâncias neutras, porém com diferentes coeficientes de partição (PACHECO et al., 2015).

Em 1941, Arne Wilhelm Kaurin Tiselius (1902-1971) acrescentou ao sistema de adsorção de Tswett uma cubeta acoplada ao final do fluxo de efluente no qual seria possível a determinação qualitativa e quantitativa dos componentes eluidos por meio de leitura ótica não humana, o que já permitia a leitura das soluções recolhidas em cubetas em um espectrofotômetro (TISELIUS, 1941).

Em 1948, Stig Melker Claesson (1917-1988) acrescentou ao sistema proposto por Tiselius um mecanismo de pressurização por um êmbolo que introduzia a fase móvel na coluna (CLAESSON, 1948).

Tiselius implementou uma classificação cromatográfica baseada em métodos distintos de eluição. O primeiro desses foi a análise frontal, em que a mistura deveria ser constantemente passada através da coluna, o segundo foi a eluição com solvente, caracterizado pelo pequeno volume da mistura na coluna acrescida de solventes puros e o terceiro era a eluição por deslocamento, em que a mistura colocada na coluna era lavada com uma solução de deslocamento (Figura 2).

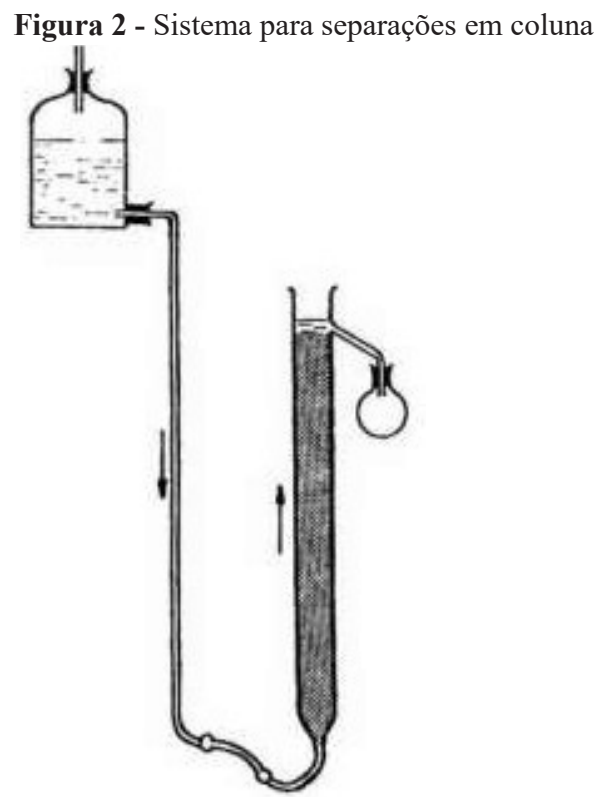

Fonte: Collins (2009)

Esta última técnica desenvolvida por Tiselius e Claesson foi aplicada para a separação de amoniácidos em colunas de troca iônica cuja eluição era obtida variando-se o pH da fase móvel (PACHECO et al., 2015).

Em 1958 surge o primeiro cromatógrafo líquido automatizado através de Willian Howard Stein (1911-1980) e Stanford Moore (1913-1982), com a participação de Darrel Heber Spackman (1925-2014) - (SPACKMAN et al., 1958). O sistema fazia eluição por gradiente de solventes, derivatização e foi capaz de analisar aminoácidos, marcando definitivamente a história da Cromatografia líquida (PACHECO et al., 2015). Não por acaso a sigla em inglês HPLC tinha, inicialmente, o significado "High Pressure Liquid Chromatograph", que depois passou a significar "High Performance Liquid Chromatograph" que, em português, tornou-se Cromatografia Líquida de AltaEficiência (CLAE).

Esta técnica passou a ser empregada para os mais diversos fins analíticos, até mesmo preparativos de substâncias altamente purificadas, necessárias a serem empregadas como padrões primários. Os cromatógrafos passaram a contar com acessórios importantes, tais como os fornos para climatizar a temperatura ideal de análise, bombas capazes de pressurizar todo o sistema até 400 bar (6000 psi) com canais individuais de solventes que permitem suas misturas ao longo da análise, abrindo um leque de polaridades diferentes e otimizando as separações, de propriedades físicas variadas e espectrógrafos eficazes inclusive para identificação de substâncias químicas através da decomposição por radicais e grupos funcionais (PACHECO et al., 2015; MAJORS, 2009).

Entretanto, a necessidade de utilização de solvente de elevada pureza e em quantidade passou a ser um problema para a cromatografia de alta eficiência quando, em 2008, veio uma grande crise pela falta de abastecimento de um dos solventes mais empregados em Cromatografia líquida, a 
Acetonitrila (LANÇAS, 2009; MAJORS, 2009). Os solventes como metanol, etanol, propanol, tetrahidrofurano e outros erampurificados a partir de produções de escala industrial, que tinham aplicações em fabricação de vários outros produtos. Somente a acetonitrila era essencialmente retirada de parte da produção de Acrilonitrila (CETESB, 2012), composto importante na fabricação de plásticos, fibras acrílicas, resinas e alguns componentes na fabricação do Nylon ${ }^{\circledR}$ (LANÇAS, 2009; EUERBY et al., 2018; MAJORS, 2009).

$\mathrm{Na}$ época algumas razões foram apontadas por conta do desabastecimento brusco. Os jogos olímpicos de 2008 realizados em Pequim acabaram por influenciar na paralização da fabricação da acrilonitrila em diversas fábricas da China, a maior produtora do mundo, naquela época, por conta da poluição que causavam. Do outro lado do mundo, com o advento do furacão Ike assolando a costa do Golfo do Texas, houve a paralização temporária de um dos principais produtores americanos, o que precipitou a fábrica a efetuar uma expansão e uma atualização do processo, estendendose a paralização da produção até o início de 2009, o que trouxe algumas dificuldades financeiras para aquela empresa (LANÇAS, 2009; MAJORS, 2009).

Em 2009 ocorreu uma queda generalizada na demanda mundial de acrilonitrila, diminuindo a disponibilidade de acetonitrila, que era seu subproduto. Um frasco de 4 litros de Acetonitrila grau HPLC chegou a ser vendido por mil dólares (MAJORS, 2009), o que gerou uma corrida por estoques do solvente e a necessidade de criar caminhos alternativos para a utilização da técnica. Entre esses caminhos se buscou a adaptação de métodos analíticos para substituir a Acetonitrila por outros solventes e várias tentativas de racionalizar o seu uso. Dentro das técnicas analíticas por HPLC, sugeriu-se que as colunas ficassem bem menores e seus "recheios" tivessem uma menor quantidade de fase estacionária, obrigando o analito a percorrê-la mais rapidamente, diminuindo os tempos de corrida, gastando menos solventes.

Porém, o equipamento tinha um limitante: o aumento da pressão, que disparava o seu desligamento automático. Este foi o gatilho para a divulgação e aceleração das vendas do UPLC, um cromatógrafo que suportava pelo menos 1000 bar (15000psi) de pressão de operação, com colunas cromatográficas menores em comprimento e diâmetro interno e partículas de sílica de núcleo sólido e superfície porosa, conforme ilustrado na figura 3 (MAJORS, 2009).
Figura 3 - Comparativo entre os tamanhos das partículas que preenchem ascolunas cromatográficas

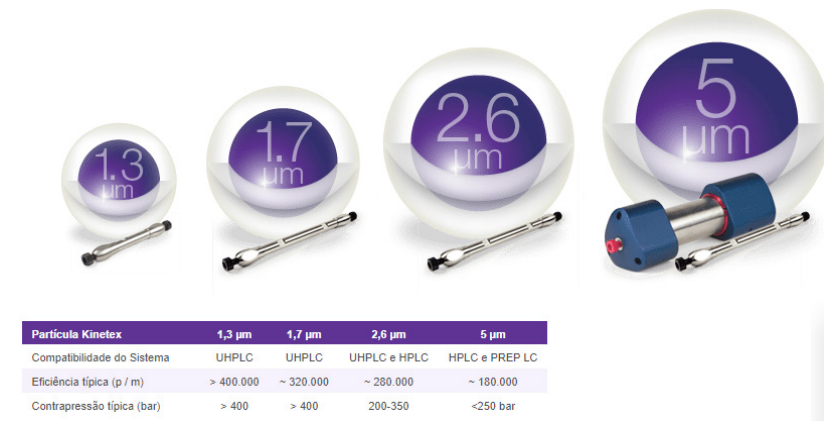

Fonte: Allcrom ${ }^{\circledR}$ Brasil.

Todas as tentativas de redução de consumo de solventes e aceleração de tempos de análise levaram à popularização do UPLC - Ultra Hight Performance LiquidChromatograph ou Cromatógrafo Líquido de Ultra Alta Performance (Figura 4).

Figura 4 - Cromatógrafo Líquido de Ultra Alta Performance

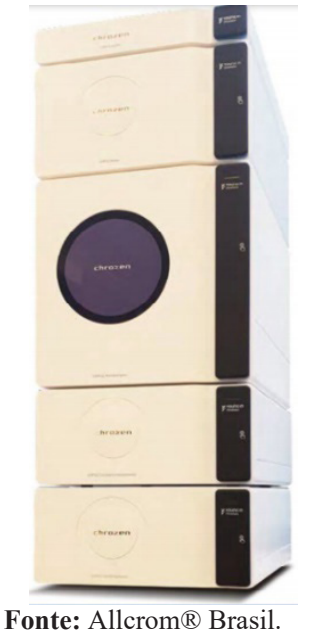

\section{Desenvolvimento}

\subsection{Material e Métodos}

Este trabalho utilizou a metodologia da pesquisa retrospectiva exploratória para reunir elementos que pudessem evidenciar a evolução da cromatografia até a técnica por UPLC, muito utilizada para análise de matérias-primas e de produtos farmacêuticos formulados. Foram consultadas as seguintes bases de dados: Pubimed, Scielo, Google academic. Buscas com aspalavras-chave: "chromatography", "history", "origin" "UPLC", "UPLC" associadas ou isoladas. O período consultado foi de 2004 a 2021. De 135 artigos no PUBMED foram selecionados apenas 2 artigos que tratam do assunto (LAFONT, 2017; BILEK and NAMIESNIK, 2016). No Google scholar de 132 artigos apenas 1 foi selecionado (GALLO and FERRANTI, 2016). Em Scielo, de 89 artigos foi selecionado 1 artigo que correspondeu ao assunto pesquisado (MALDANER and JARDIM, 2009). 


\section{Resultados e Discussão}

Em 2004, surgiu o UPLC, da empresa Waters (WATERS $®$ CORPORATION, 2020), com a promessa de reduzir tempo de análise, diminuir gastos com reagentes e substituir o uso dos HPLCs (MAJORS, 2009). Entretanto, muitas barreiras técnicas precisavam ser resolvidas. Na época do lançamento do UPLC havia menos funcionalidades oferecidas em relação aos HPLCs, pois se tratava de bombas binárias, ou seja: capazes de misturar até dois solventes diferentes em proporções prédefinidas pelo usuário (contra as ternárias - mistura de três solventes - e quaternárias - mistura de 4 solventes diferentes - de HPLC), com um compartimento de coluna (os HPLCs já ofereciam compartimentos múltiplos e uma variedade de recheios de coluna). Além da comparação de preços entre os dois tipos de equipamentos. O primeiro custava em torno de US\$60000,00, já o UPLC entre US\$130000,00 e US\$170000,00 (cerca de duas ou três vezes mais caros na época) inviabilizava a aquisição em maior escala.

A utilização da nova técnica advinha da implementação regulatória das alterações do novo sistema (LANÇAS, 2009; MAJORS, 2009). Os órgãos regulatórios exigiam avaliação técnica de todas as implementações analíticas nos laboratórios, como: seguir rigorosamente as técnicas já publicadas e descritas nos compêndios oficiais (as farmacopeias), atender às limitações de alterações analíticas permitidas, como, limitação paraalterar o tamanho de colunas cromatográficas, alterar o tempo de retenção dos analitos e proporções descritas de fase móvel, mudança de equipamento (e até mesmo a sala utilizada deveria ser qualificada), o que envolvia aumento de custo e tempo (LANÇAS, 2009; EUERBY et al., 2018; MAJORS, 2009). Novas validações ou co-validações deveriam ser, segundo diretrizes do FDA - Food and Drug Administration - que declarou em meados de 2009, no auge da escassez da Acetonitrila, "independentemente das mudanças que uma empresa faça para lidar com a escassez (de Acetonitrila), a validação do método apropriado e a conformidade com as boas práticas de fabricação relevantes (CGMPs - Current GoodManufacturing Procedures) são necessárias" (LANÇAS, 2009; EUERBY et al., 2018; MAJORS, 2009). Em 2009, os laboratórios farmacêuticos assumiram a necessidade de diminuir o consumo de Acetonitrila, em função do tempo de escassez que iria perdurar. Até 2020 a empresa Waters ${ }^{\circledR}$ possuía 70000 equipamentos instalados no mundo (WATERS ${ }^{\circledR}$ CORPORATION, 2020). Portanto, na medida em que os UPLCs foram ganhando espaço, foram sendo colocados lado a lado com os HPLCs nas bancadas, já que com o uso do UPLC esperava-se maior produtividade com menor custo.

Comparando-se as duas técnicas analíticas, HPLC e UPLC, a primeira requer mais tempo de análise. Por exemplo, uma análise de Cefuroxima (corrida de um padrão e uma amostra) leva 80 minutos no HPLC é reduzida para 10 min no UPLC. Considerando um fluxo de $1 \mathrm{~mL} /$ minuto de fase móvel na análise por HPLC, são gastos $80 \mathrm{~mL}$ de fase móvel enquanto no UPLC se utiliza um fluxo de $0,1 \mathrm{~mL} /$ minuto de fase móvel, consumindo $10 \mathrm{~mL}$ de fase móvel por análise - corrida de padrão e amostra - (PORTO, 2014). Além da economia de fase móvel e de tempo há um aumento de produtividade. Isso quer dizer que, além da economia de fase móvel, o aumento da produtividade analítica é capaz de justificar, com certa facilidade, que um único sistema de UPLC pode fazer o trabalho de 2 ou 3 sistemas de HPLC, o que significa menor consumo de solventes, aumento da produtividade, diminuição dos custos de manutenção, economia de mão de obra especializada, incluindo as qualificações periódicas de performance e operação e o aumento da velocidade na liberação de produtos, tanto para a produção, quanto para a venda de lotes de fabricação.

Além disso, os testes de validação de limpeza, validação de processos e validação de métodos analíticos são reduzidos a um terço, diminuindo, consideravelmente, os tempos de parada de máquinas e processos produtivos, resolvendo rapidamente possíveis falhas nos testes de adequação de sistemas SST - System Suitability Test - (LANÇAS, 2009). Em contrapartida, quando há necessidade de manutenção desses equipamentos, a sua parada representa perda significativa de produtividade, face a sua grande lista de amostras de rotina, interferindo na velocidade de liberação de resultados. Por esse motivo os laboratórios ainda não renunciam aos sistemas de HPLC. Outra limitação da técnica é que compêndios oficiais ainda descrevem técnicas por HPLC, que não podem ser substituídas por outras técnicas por questões de patentes ou outras questões de regulamentações técnicas legais. Existem casos que, em função da complexidade de certas amostras não foi possível desenvolver uma técnica por UPLC. Um exemplo são os produtos naturais, cujo extrato seco ou hidroalcóolico é constituído por misturas complexas inviabilizando as análises por UPLC, cujas colunas são de diâmetros reduzidos e por "recheio" de partículas diminutas que seriam imediatamente inutilizadas por entupimentos e retenções irreversíveis (MAJORS, 2009; PINTO, 2020).

Entre os vários trabalhos de migração de método cromatográfico por HPLC para UPLC de sucesso há o trabalho de Porto (PORTO, 2014), no qual ele utilizou Cefuroxima, que mostra a maior eficiência e economia de tempo e reagentes. Sendo a Cefuroxima uma molécula polar, é preciso que a fase móvel seja mais polar que a fase estacionária, ou seja, operar em fase reversa. Nos Quadros 1 e 2 se observa os comparativos da redução de tempo analítico e consumo de reagentes. 
Quadro 1 - Condições cromatográficas de método analítico de Cefuroxima em Cromatografia líquida - HPLC

\begin{tabular}{|c|c|c|c|}
\hline Parâmetro & \multicolumn{3}{|c|}{ Descrição } \\
\hline Coluna & \multicolumn{3}{|c|}{ 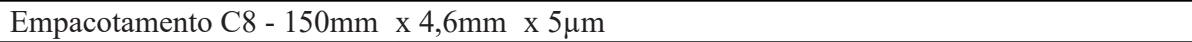 } \\
\hline Fase móvel A & \multicolumn{3}{|c|}{$\begin{array}{l}\text { Solução tampão pH 3,5: Dissolva } 680 \mathrm{mg} \text { de Acetato de Sódio tri-hidratado e } 5,7 \mathrm{~mL} \text { de Ácido } \\
\text { Acético Glacial 100\% num balão de } 1000 \mathrm{~mL} \text { com água purificada. Ajuste o pH para 3,5 com } \\
\text { Ácido Acético Glacial 100\% ou solução de Hidróxido de Sódio } 1 \mathrm{M} \text {. }\end{array}$} \\
\hline Fase móvel B & \multicolumn{3}{|c|}{ Acetonitrila } \\
\hline $\begin{array}{c}\text { Volume de } \\
\text { injeção }\end{array}$ & \multicolumn{3}{|l|}{$10 \mu \mathrm{L}$} \\
\hline Fluxo & \multicolumn{3}{|l|}{$1,5 \mathrm{~mL} / \mathrm{min}$} \\
\hline $\begin{array}{c}\text { Detector } \\
\text { PDA/UV-Vis } \\
\end{array}$ & \multicolumn{3}{|l|}{$\Lambda=273 \mathrm{~nm}$} \\
\hline $\begin{array}{l}\text { Temperatura } \\
\text { das amostras }\end{array}$ & \multicolumn{3}{|l|}{$8^{\circ} \mathrm{C}$} \\
\hline $\begin{array}{c}\text { Temperatura } \\
\text { da Coluna }\end{array}$ & \multicolumn{3}{|l|}{$35^{\circ} \mathrm{C}$} \\
\hline \multirow{10}{*}{ Gradiente } & Tempo(min) & $\begin{array}{c}\text { \%Fasemóvel } \\
\text { A }\end{array}$ & $\begin{array}{c}\text { \%Fasemóvel } \\
\text { B }\end{array}$ \\
\hline & 0 & 93 & 7 \\
\hline & 5 & 93 & 7 \\
\hline & 9 & 88 & 12 \\
\hline & 18 & 88 & 12 \\
\hline & 20 & 80 & 20 \\
\hline & 25 & 80 & 20 \\
\hline & 32 & 63 & 40 \\
\hline & 33 & 93 & 7 \\
\hline & 40 & 93 & 7 \\
\hline
\end{tabular}

*1 = PDA: Sigla que vem do inglês "Photo Diode Array", tipo de detector capaz de fazer uma varredura de absorbâncias em vários comprimentos de onda ao mesmo tempo. E ele pode executar essa tarefa na faixa do UV (Ultra-violeta) e do VIS (Visível).

Fonte: Porto (2014).

Nota-se no Quadro 1 o grau de complexidade para a separação dos componentes daamostra em função da tabela de gradiente, variando ao longo do tempo de corrida as porcentagens de misturas entre duas fases móveis. Este recurso é necessário, em função da necessidade da variação da polaridade da fase móvel ao longo da análise.

Quadro 2 - Condições cromatográficas de validação de método analítico de doseamento de princípio ativo Cefuroxima em Cromatografia de Ultra eficiência - UPLC

\begin{tabular}{|c|c|c|c|}
\hline Parâmetro & \multicolumn{3}{|c|}{ Descrição } \\
\hline Coluna & \multicolumn{3}{|c|}{$\begin{array}{l}\text { Empacotamento BEH (Ethylene Bridged Hybrid } \\
\text { - Partícula Híbrida componte de etileno) } \mathrm{C} 8-100 \mathrm{~mm} \times 2,7 \mathrm{~mm} \times \\
1,7 \mu \mathrm{m}\end{array}$} \\
\hline Fase móvel A & \multicolumn{3}{|c|}{$\begin{array}{l}\text { Solução tampão pH 3,5: Dissolva } 680 \mathrm{mg} \text { de Acetato de Sódio tri-hidratado e } 5,7 \mathrm{~mL} \text { de } \\
\text { Ácido Acético Glacial } 100 \% \text { num balão de } 1000 \mathrm{~mL} \text { com água purificada. Ajuste o pH para } \\
3,5 \text { com Ácido Acético Glacial } 100 \% \text { ou solução de Hidróxido de Sódio } 1 \mathrm{M} \text {. }\end{array}$} \\
\hline Fase móvel B & \multicolumn{3}{|l|}{ Acetonitrila } \\
\hline $\begin{array}{l}\text { Volume de } \\
\text { injeção }\end{array}$ & \multicolumn{3}{|l|}{$1 \mu \mathrm{L}$} \\
\hline Fluxo & \multicolumn{3}{|l|}{$0,5 \mathrm{~mL} / \mathrm{min}$} \\
\hline $\begin{array}{c}\text { Detector } \\
\text { PDA/UV-Vis"1 }\end{array}$ & \multicolumn{3}{|l|}{$\Lambda=273 \mathrm{~nm}$} \\
\hline Temperatura das amostras & \multicolumn{3}{|l|}{$8^{\circ} \mathrm{C}$} \\
\hline $\begin{array}{r}\text { Temperatura } \\
\text { da coluna }\end{array}$ & \multicolumn{3}{|l|}{$35^{\circ} \mathrm{C}$} \\
\hline $\begin{array}{r}\text { Concentração } \\
\text { de trabalho }\end{array}$ & \multicolumn{3}{|l|}{$0,1 \mathrm{mg} / \mathrm{mL}$} \\
\hline \multirow{2}{*}{$\begin{array}{r}\text { Gradiente } \\
\text { (nãoaplicável) }\end{array}$} & Tempo(min) & $\begin{array}{c}\text { \%Fasemóvel } \\
\text { A }\end{array}$ & $\begin{array}{c}\text { \%Fasemóvel } \\
\text { B }\end{array}$ \\
\hline & 5 & 88 & 12 \\
\hline
\end{tabular}

Fonte: Porto (2014). 


\section{Conclusão}

Após 16 anos de desenvolvimento tecnológico, os sistemas de UPLC (UHPLC) atingiram todas as potencialidades analíticas de recursos melhorados, com vantagens substanciais de economia de solventes e de tempo analítico e pode ser considerado um substituto para a maioria dos equipamentos de HPLC existentes em laboratórios de controle de qualidade, desenvolvimento analítico e estudos de estabilidade. Restarão, para o HPLC, as técnicas analíticas de misturas complexas de plantas, extratos e outros produtos naturais complexos.

As vantagens do uso do UPLC, portanto, superam as desvantagens da sua utilização, principalmente, por conta da economia de solventes, cuja quantidade é reduzida de forma significativa, diminuição de resíduos a serem descartados, contribuindo na preservação do meio ambiente, o tempo de resposta e liberação de lotes de produtos nos laboratórios aumentando a produtividade e a otimização da mão de obra especializada empregada nas análises.

\section{Referências}

ALLCROM ${ }^{\circledR}$ Brasil - Representante da marca sul-coreana Young Lin ${ }^{\circledR}$ noBrasil. Disponível em: https://www.allcrom.com. br/produto/UPLC-cromatografo- liquido-de-ultra-alta-pressaochrozen/. Acesso em: 13 dez. 2020.

BILEK, M.; NAMIEŚNIK, J. Chromatography techniques in pharmaceutical analysis in poiand: history and the presence on the basis of papers published in selected polish Pharmaceutical Journals in XX Century. Acta Pol. Pharm., v.73, n.3, p.605-612, 2016. doi: 10.1016/j.anplas.2017.08.002.

CETESB - Divisão de Toxicologia Humana e Saúde Ambiental. Ficha de Informação Toxicológica de Acrilonitrila. Jul. 2012.

LANÇAS, F.M. Como economizar (ou eliminar o uso de) acetonitrila em tempos de "crise"? São Carlos: USP, 2009.

MALDANER, L.C.; JARDIM, I.C.S.F. O estado da arte da cromatografia líquida de ultra eficiência. Quím. Nova, v.32, n.1, p.214-222, 2009. doi: 10.1590/S0100-40422009000100036.

CLAESSON, S. Frontal analysis and displacement development in chromatography. Ann. New York Acad. Scie., v.49, p 183204, 1948. doi: 10.1111/j.1749-6632.1948.tb35249.

COLLINS, C.H.I. Michael Tswett e o "nascimento" da Cromatografia. Rev. Scie. Chromatographica, v.1, n.1, p.7-20, 2009.

EUERBY, M.R.; SCHAD, G.J. Shimadzu Europa GmbH. AlbertHahn Strasse n.6-10, 47269 Duisburg, Germany, 2018.
GALLO, M.; FERRANTI, P. The evolution of analytical chemistry methods in foodomics. J. Chromatography A, v.1428, p.3- 15, 2016. doi:10.1021/jf900189s.

LAFONT, O. Les origines de l'analyse et du contrôle des médicaments [The origins of analysis and of drugs control]. Ann. Pharm. Fr., v.75, n.4, p.302-308, 2017. doi: 10.1016/j. pharma.2017.02.003.

MAJORS, R.E. The Continuing Acetonitrile Shortage: How to Combat it or Livewith It. 2009. Disponível

em: https://www.chromatographyonline.com/view/continuingacetonitrile-shortage-how-combat-it-or-live-it. Acesso em 12 dez. 2020.

MALDANER, L.; JARDIM, I. C. S. F. O estado da arte da cromatografia líquida de ultra eficiência. Departamento de Química Analítica, Instituto de Química, Universidade Estadual de Campinas, CP 6154, 13084-971 Campinas - SP, Brasil. Quim. Nova, v. 32, n. 1, p.214-222, 2009. doi: 10.1590/S010040422011000500020.

MARTIN, A.J.P.; SYNGE, R.L.M. A New form of chromatogram employing two liquid phases. Biochem J., v. 35, n.12, p.13581368, 1941. doi: 10.1042/bj0351358.

MORETTO, L.D. Sindusfarma: 80 anos. Guardião da História da Indústria Farmacêutica no Brasil. São Paulo: Sindusfarma, 2013.

MÜHLEN, C.V.; LANÇAS, F.M. Cromatografia unificada. Universidade de São Paulo, Instituto de Química de São Carlos. Quim. Nova, v.27, n.5, p.747-753, 2004. doi: 10.4322/ sc.2013.009.

PACHECO, A.S. et al. História da Cromatografia Líquida. Rev. Virtual Quim., v.7, n.4, p. 1225-1271, 2015. doi: 10.5935/19846835.20150069.

PHENOMENEX ${ }^{\circledR}$ Corporation - Blog: Science Unfiltered. Disponível em: https://phenomenex.blog/category/sciencenews/. Acesso em 07 abr. 2021.

PINTO, G.M.F. Vale a pena migrar de HPLC para UPLC? 2020. Disponível em: https://cromvallab.com/2020/03/11/vale-a-penamigrar-de-hplc-para-uplc/ . Acessoem 14 dez. 2020.

PORTO, H.S.M. Avaliação de aspectos críticos à transferência e validação de métodos analíticos. Portugal: Universidade de Coimbra, 2014.

SPACKMAN, D.H.; STEIN, W.H.; MOORE, S. Automatic Recording Apparatus for Use in the Chromatography of Amino Acids. Anal. Chem., v. 30, n.7, p.1190-1206, 1958. doi: 10.1021/ ac60139a006.

TISELIUS, A. A new procedure for adsorption analysis. Science, v.94, p.145-146, 1941. doi: 10.1126/science.94.2432.145.

WATERS CORPORATION. Folder:Alliance ${ }^{\circledR}$ HPLC. Disponível em: https://www.waters.com/waters/Alliance-HPLC- System/ nav.htm?cid $=534293 \&$ alias $=$ Alias_Alliance_INS\&locale $=$ pt_ BR. Acesso em 13 dez. 2020. 